\title{
ORIGINAL RESEARCH \\ Is CT Still Useful in the Study Protocol of Retinoblastoma?
}

P. Galluzzi

T. Hadjistilianou

A. Cerase

S. De Francesco

P. Toti

C. Venturi

BACKGROUND AND PURPOSE: Intralesional calcium deposition is considered a key element for differentiating retinoblastoma from simulating lesions. Our aim was to assess whether MR imaging associated with ophthalmologic investigations (ophthalmoscopy and ultrasonography) could replace CT in the detection of diagnostic intralesional calcifications in retinoblastoma.

\begin{abstract}
MATERIALS AND METHODS: Ophthalmoscopic findings, MR images, CT scans, and histologic examination of 28 retinoblastomas from 23 consecutive children $(11$ males, 12 females; age range at admission, 1-35 months; mean age, 11 months; median age, 9 months) were retrospectively evaluated. Ultrasonography was performed in 18 patients with 21 retinoblastomas. MR imaging included T2-weighted spin-echo and gradient-echo images, fluid-attenuated inversion recovery images, and T1-weighted spin-echo images with and without contrast enhancement. Clinical data were integrated with MR imaging data to evaluate the utility of both approaches to discover calcifications; particularly, a correlation between intralesional signal-intensity void spots on MR imaging and hyperattenuating areas on CT scans was performed.
\end{abstract}

RESULTS: Ophthalmoscopy detected calcifications in 12 of 28 eyes $(42.85 \%)$. Ultrasonography detected calcifications in 20 of 21 eyes $(95.23 \%)$. CT showed hyperattenuating intralesional areas consistent with calcifications in 27 of 28 eyes (96.42\%). MR imaging showed intralesional signalintensity void spots in 25 of 28 eyes (89.28\%). All spots detected with MR imaging matched the presence of calcifications on CT scans. Gradient-echo T2*-weighted and fast spin-echo T2-weighted images showed the highest degree of correlation with CT. When we put together ophthalmoscopy, ultrasonography, and MR imaging data, no calcifications detected on CT were missed, and the differential diagnosis was thorough.

CONCLUSIONS: A combination of clinical data and MR images may remove potentially harmful ionizing radiation from the study protocol of retinoblastoma.

$E_{p}^{*}$ arly diagnosis and treatment of retinoblastoma greatly improve the patient's survival rate and quality of vision. Because fine-needle aspiration biopsy carries a significant risk of procedure-related tumor seeding, ${ }^{1,2}$ patients with retinoblastoma are treated on the basis of clinical and imaging data, without a histologic diagnosis. Thus, noninvasive diagnostic accuracy is crucial. A decisive additional support of the ophthalmoscopic diagnosis is given by matching intralesional calcifications. ${ }^{3,4}$ The detection of intraocular calcium deposition is a key element to differentiate retinoblastoma from simulating lesions. Histology reports show that retinoblastomas calcify in $\leq 95 \%$ of cases $^{5}$; CT has a designated sensitivity in detecting calcifications in retinoblastoma of $81 \%-96 \%,{ }^{6-10}$ whereas the sensitivity of sonography has been reported to be $\leq 92.5 \%$ of cases. ${ }^{4}$ In children younger than 3 years of age, an intrabulbar calcified mass is most likely a retinoblastoma. ${ }^{11,12}$ However, in children older than 3 years of age, other intraocular lesions, such as retinopathy of prematurity, toxocariasis, Coats' disease, retinal astrocytoma, and optic nerve drusen may appear as a calcified masses. ${ }^{3,10}$ Retinoblastoma may very

\section{Received March 6, 2009; accepted after revision April 27}

From the Unit of Diagnostic and Therapeutic Neuroradiology (P.G, A.C., C.V.), Azienda Ospedaliera Universitaria Senese, Siena, Italy; InterDepartmental Center of Nuclear Magnetic Resonance (P.G, A.C., C.V.), Policlinico "Le Scotte," Siena, Italy; and Unit of Ophthalmology (T.H., S.D.F.) and Department of Human Pathology and Oncology (P.T.), University of Siena, Siena, Italy.

Please address correspondence to Paolo Galluzzi, MD, Unit of Diagnostic and Therapeutic Neuroradiology, Azienda Ospedaliera e Universitaria Senese, Policlinico "Le Scotte," Viale Bracci 1, 53100 Siena, Italy; e-mail: galluzzip@libero.it

DOI 10.3174/ajnr.A1716 rarely occur in older patients, but we have had a new diagnosis in a child older than 15 years of age (personal observation). ${ }^{13}$

CT or MR imaging or both are usually required to detect optic nerve, choroid, and scleral infiltration stages; disease spread beyond the eye; or intracranial disease. Imaging studies are used for diagnostic purposes only in very selected instances, as in cases in which ophthalmoscopy is impossible, such as with the presence of opaque ocular refractive media and massive retinal detachment or for differentiating lesions simulating retinoblastoma. ${ }^{11,14-23}$

The purpose of this study was to assess the utility of the association of ophthalmologic evaluation and MR imaging in the detection of intralesional calcifications in retinoblastoma, to evaluate whether CT is still mandatory in the protocol study of retinoblastoma and simulating lesions.

\section{Materials and Methods}

The population of this prospective study included 23 consecutive children (11 males, 12 females). Patients' ages ranged from 1 to 35 months (mean age, 11 months; median age, 9 months). Fifteen patients had unilateral retinoblastoma, and the remaining 8 had bilateral retinoblastoma. Patients' clinical data were collected from inpatient and outpatient medical records of the referral center for retinoblastoma of our institution, including sex, age, affected eyes, and ophthalmoscopic data. Ultrasonographic data were available in 18 patients with 21 affected eyes. Three patients with bilateral retinoblastoma had already been submitted to enucleation of 1 eye before the admission to the department of ophthalmology. Parents gave informed consent for MR imaging and CT scanning in all the cases. The 


\begin{tabular}{|c|c|c|c|c|c|}
\hline \multicolumn{6}{|c|}{ Correspondence between signal-void spots on MR imaging and calcifications on CT } \\
\hline Sequence & True-Positive (\%) & True-Negative (\%) & False-Positive & False-Negative (\%) & Uncertain $(\%)$ \\
\hline SE T1 & $13 / 28(46.42)$ & $1(3.57)$ & & $7(25)$ & $7(25)$ \\
\hline TSE T2 & $17 / 28(60.71)$ & $1(3.57)$ & & $5(17.85)$ & $5(17.85)$ \\
\hline FS FLAIR & 15/22 (68.18) & $1(4.54)$ & & $4(18.18)$ & $2(9.09)$ \\
\hline GE T2* & $21 / 24(87.5)$ & $1(4.16)$ & & $3(12.5)$ & \\
\hline 3D TSE T2 & $10 / 10(100)$ & & & & \\
\hline 3D GE T2* & $4 / 4(100)$ & & & & \\
\hline SE T1 gd & $11 / 20(55)$ & & & $5(25)$ & $4(20)$ \\
\hline FS SE T1 gd & $9 / 19(47.36)$ & $1(5.26)$ & & $5(25)$ & $4(20)$ \\
\hline
\end{tabular}

Note:-SE indicates spin-echo; GE, gradient-echo; TSE, turbo-spin-echo; FS, fat-suppressed; FLAIR, fluid-attenuated inversion recovery; gd, gadolinium.

study protocol was approved by the ethics committee of our institution.

Two experienced ophthalmologists (T.H., S.D.F.) performed ophthalmoscopy, which always included careful examination of the ocular fundus and a search for intralesional calcifications.

MR imaging of the orbit was performed on a $0.5 \mathrm{~T}$ unit (Gyroscan NT5; Philips Medical Systems, Best, the Netherlands) in 2 patients with 3 retinoblastomas and on a 1.5T unit (Gyroscan ACS-NT15; Philips Medical Systems) in 21 patients with 25 retinoblastomas.

In both patients studied on the $0.5 \mathrm{~T}$ unit, unenhanced transverse spin-echo T1-weighted images (TR, $500 \mathrm{~ms}$; TE, $25 \mathrm{~ms}$; thickness, 3 $\mathrm{mm}$; NEX, 2) and transverse fast-field echo $\mathrm{T} 2^{\star}$ gradient-echo images (TR, $415 \mathrm{~ms}$; TE, $27 \mathrm{~ms}$; thickness, $3 \mathrm{~mm}$; flip angle, 15 ; NEX, 3) were obtained; in 1 patient, sagittal spin-echo T1-weighted images after gadolinium injection (TR, $500 \mathrm{~ms}$; TE, $25 \mathrm{~ms}$; thickness, $3 \mathrm{~mm}$; NEX, 2) were obtained.

In the patients studied on the $1.5 \mathrm{~T}$ unit, unenhanced transverse spin-echo T1-weighted images (TR, $500 \mathrm{~ms}$; TE, $20 \mathrm{~ms}$; thickness, 3.3 $\mathrm{mm}$; NEX, 2) and transverse turbo spin-echo (TSE) T2-weighted images (TR, $4000 \mathrm{~ms}$; TE, $120 \mathrm{~ms}$; echo-train length, 11; thickness, 3 $\mathrm{mm}$; NEX, 2) were obtained in all of them (25 eyes). Unenhanced transverse fast-field echo T2* gradient-echo images (TR, $376 \mathrm{~ms}$; TE, $23 \mathrm{~ms}$; thickness, $3.3 \mathrm{~mm}$; flip angle, $15^{\circ}$; NEX, 3), transverse fatsuppressed turbo fluid-attenuated inversion recovery images (TR, 5000 ms; TE, 100 ms; TI, 2000 ms; echo-train length, 18; thickness, 2.2 mm; NEX, 3), transverse 3D TSE T2-weighted images (3D TSE T2) (TR, $3000 \mathrm{~ms}$; TE, $150 \mathrm{~ms}$; echo-train-length, 17; thickness, $1.5 \mathrm{~mm}$; gap, $-0.8 \mathrm{~mm}$; NEX, 1), and transverse $3 \mathrm{D}$ fast-field echo T2* gradient-echo images (TR, $80 \mathrm{~ms}$; TE, $23 \mathrm{~ms}$; thickness, $2 \mathrm{~mm}$; gap, $-1 \mathrm{~mm}$; flip angle, $15^{\circ}$; NEX, 2) were obtained in 19 patients (21 eyes), 18 patients (22 eyes), 10 patients (10 eyes), and 4 patients (4 eyes), respectively. After intravenous injection of gadolinium, transverse spin-echo T1-weighted images (TR, $500 \mathrm{~ms}$; TE, $20 \mathrm{~ms}$; thickness, $3.3 \mathrm{~mm}$; NEX, 2) and transverse fat-suppressed spin-echo T1weighted images (TR, $550 \mathrm{~ms}$; TE, 14 ms; thickness, 3.3. mm; NEX, 2) were obtained in 15 patients (19 eyes).

In all patients, orbit examination was performed with a surface coil (used as a receiver coil) positioned over both orbits, parallel to the coronal plane. In most patients, MR imaging was performed by using chloral hydrate for sedation (100 mg/kg of body weight).

MR images were qualitatively evaluated for evidence of intralesional signal-intensity-void spots by 2 experienced neuroradiologists (P.G., A.C.), unaware of ophthalmologic, CT, and histopathologic results. Intralesional spots were recorded as present, absent, or uncertain. In cases of initial disagreement, the final decision was made by consensus.

CT scans of the orbits were obtained in all 23 patients (Sytec and BrightSpeed; GE Healthcare, Milwaukee, Wis). Unenhanced CT of the orbits was performed in all 23 patients with 28 retinoblastomas. Contiguous 1.25 - to $1.5-\mathrm{mm}$-thick sections parallel to the canthomeatal line through the orbits were obtained. An experienced neuroradiologist (C.V.), blinded to MR imaging, ophthalmologic, and histologic findings, examined the CT scans to evaluate the presence of hyperattenuating intraocular areas consistent with calcifications. Correspondence between intraocular intralesional spots on MR imaging and intraocular hyperattenuating areas on CT was then evaluated.

Histologic examination was performed in all 28 enucleated eyes. The eyes were fixed in saline-buffered formalin, sampled, embedded in paraffin, sectioned, and stained with hematoxylin-eosin.

\section{Results}

In all the enucleated eyes, the diagnosis of retinoblastoma was histologically confirmed, with evidence of calcifications in 27 of 28 eyes.

Calcifications were ophthalmoscopically detected in 12 of 28 eyes $(42.85 \%)$. Ultrasonography showed posterior shadowing, consistent with calcifications, in 20 of 21 eyes (95.23\%). CT showed hyperattenuating intralesional areas consistent with calcifications in 27 of 28 eyes (96.42\%). Comparison between signal-intensity voids detected on each MR image and calcifications on CT is summarized in the Table.

3D TSE T2-weighted and 3D gradient-echo T2*-weighted images showed the highest specificity (100\%), followed by 3.3mm-thick gradient-echo T2* images $(87.5 \%)$. When all 3 sequences were performed, there was $100 \%$ correspondence between signal-intensity voids on MR images and calcifications on CT scans.

\section{Discussion}

Accurate and expedient diagnosis of retinoblastoma by a careful ophthalmoscopic examination through clear optical media is usually quite easy. ${ }^{24}$ When refractive media are clear, the presence of large calcified areas is also easily documented by ophthalmoscopy.

Sonography is performed to assess the presence of vitreal or subretinal masses, the configuration and acoustic reflectivity of such masses, and the possible presence of retinal detachment. Sonography may be also used to detect the presence of intraocular calcifications successfully; particularly, calcium has been detected in $\leq 92.5 \%{ }^{4}$ of cases in the most frequent retinoblastoma forms of growth $(\leq 95.23 \%$ in our personal records). However, sonographic capacity for detecting small calcified masses is limited by the presence of complex intraocular interfaces associated with vitreous opacities, retinal masses, subretinal fluid, and retinal detachment. 

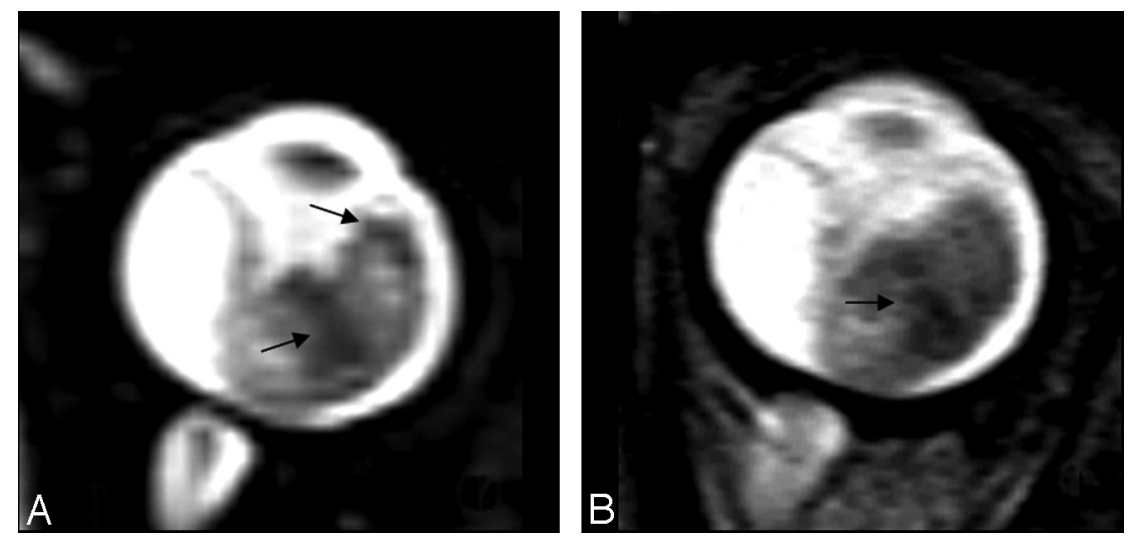

Fig 1. Comparison of intralesional hypointensity (arrows) on 3D TSE T2 $(A)$ and TSE T2 (B).

Studies such as CT and MR imaging provide valuable help in the differential diagnosis between retinoblastoma and the simulating lesions, also supplying a detailed study of intraand extraocular structures (including the brain). Moreover, they are not impaired by those conditions that weaken sonography and ophthalmoscopic examinations.

CT and MR imaging represent the primary modalities for visual-pathway imaging in children. MR imaging is the method of choice in depicting ocular lesions; ${ }^{811,25-27}$ however, CT is still the method of choice for detecting intraocular calcium and investigating orbital pathologies. ${ }^{28,29}$

The disadvantages of CT are reduced soft-tissue contrast and spatial resolution compared with MR imaging; the presence of the potential risk of iodinated contrast administration; and radiation exposure, especially in those patients with the hereditary form of the disease, who have elevated radiosensitivity. MR imaging is considered the method of choice for follow-up imaging in already diagnosed cases, especially to detect preclinical intracranial localization of the neoplastic disease. $^{30}$ The MR imaging appearance of retinoblastoma is usually specific enough to differentiate retinoblastoma from simulating lesions ${ }^{11}$; however, currently, MR imaging is not considered as specific as CT in detecting intraocular calcification.

The effect of calcium on tissue signal intensity in MR imaging is variable: ${ }^{31,32}$ calcified tissues are far more frequently hypointense (due to diamagnetic properties of the calcium), ${ }^{33}$ but isointensity or hypointensity is also possible, depending on the concentration of the surface area of the calcium particles and on the MR imaging technique used. Isohyperintensity has been shown in particles with $\leq 30 \%$ calcium by weight, but it decreases at high concentrations, as in the retinoblastomaassociated calcifications.

On spin-echo T2-weighted images, detectable calcifications are far more frequently hypointense, especially when a high-field magnet and thin sections are used. One of the deficiencies of the spin-echo sequence lies in its well-recognized limited ability to detect calcification consistently.

Recent developments have introduced a very high-resolution 3D TSE T2, which allows very thin sections with high signal intensity-to-noise ratios: though this sequence allows a more accurate detection of orbital pathology, it could be more prone to motion artifacts than a conventional multisection sequence $^{34}$ and, to the best of our knowledge, the usefulness of such a sequence in calcium detection has not been demonstrated. In our study group, the 3D TSE T2 sequence showed higher specificity in showing intralesional spots than the conventional fast spin-echo T2-weighted sequence (Fig 1).

Gradient-echo $\mathrm{T} 2{ }^{*}$-weighted imaging has been shown to be more effective than spin-echo techniques in the detection of calcified structures, typically as very hypointense regions (Fig 2). ${ }^{35,36}$ Because retinoblastoma is a heterogeneous tumor, the partially calcified areas may appear as hypointense foci within the higher intensity of the soft-tissue mass (Fig 3).

In our study, this behavior has been a significant advantage: the higher degree of signal-intensity loss of the calcifications and the lower hypointensity of the whole tumor on gradientecho $\mathrm{T} 2{ }^{*}$-weighted images in comparison with routine TSE T2-weighted spin-echo images, has led to a superior detectability of intralesional spots in the former sequence; moreover, $3 \mathrm{D}$ gradient-echo $\mathrm{T} 2^{*}$-weighted images increase the sensibility in intralesional signal intensity-loss spot detection (Fig 4).

Intralesional spots were detectable in 27 of the 28 eyes examined. Among these 27 eyes, in 2 patients, intralesional spots were detectable only on 3D TSE T2 sequences, whereas in 3 patients, they were detected only on gradient-echo $\mathrm{T}^{*}$ weighted imaging. Putting together 3 -mm-thick gradientecho $\mathrm{T} 22^{*}$, tridimensional TSE $\mathrm{T} 2$, and tridimensional gradient-echo $\mathrm{T} 2^{\star}$-weighted images, we found a correspondence of $100 \%$ between the presence of intralesional spots on MR imaging and the presence of calcifications on CT examinations. Even fine calcifications were detectable (Fig 5).

The criterion for calcifications as a localized marked hypointense signal intensity on both T1- and T2-weighted images without contrast enhancement has been sporadically described. Mihara et $\mathrm{a}^{37}$ found spots of round signal-intensity drop-off in 3 of 7 retinoblastomas, considered calcifications on CT; recently, Lemke et $\mathrm{al}^{7}$ showed MR imaging high sensitivity $(91.7 \%)$ and specificity (88.9\%) in detecting a marked amount of intraocular calcifications. Our series confirms the results of Lemke et al, adding the chance to reach $100 \%$ correspondence by using appropriate sequences and coupling neuroradiologic and clinical findings.

A limitation of our study was that the appearance of calcification at T2-weighted images is not specific: Actually, aside from calcification, hypointensity on T2-weighted images can be found at the boundaries or inside the substance of various stages of hemorrhage (ie, deoxyhemoglobin, methemoglobin, 

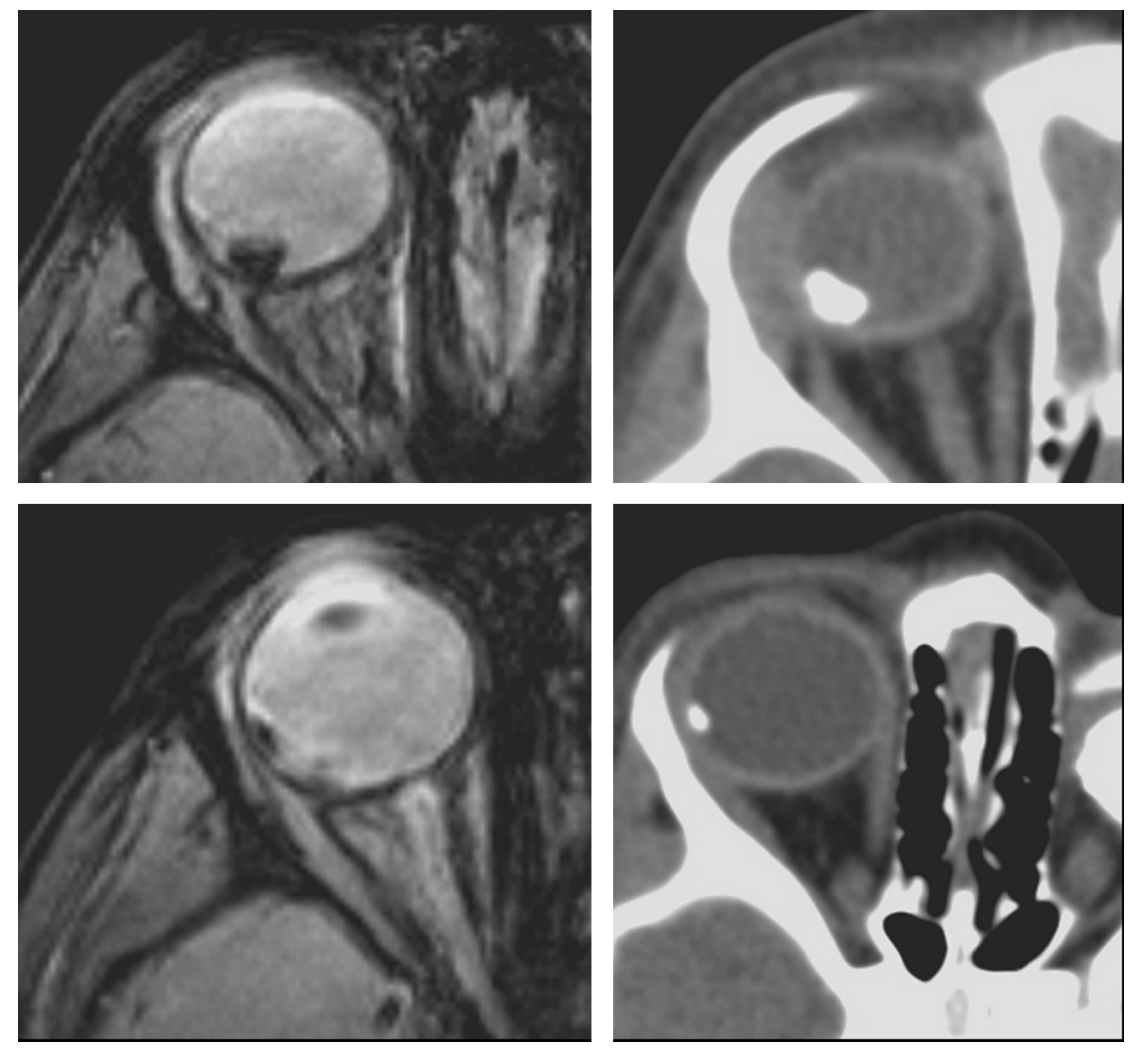

Fig 2. Conventional 3.3-mm-thick gradient-echo $\mathrm{T} 2^{*}$-weighted images (on the left) and CT scans (on the right) of the same patient. Note the correspondence between hypointense spots on MR imaging and hyperattenuating spots on CT.
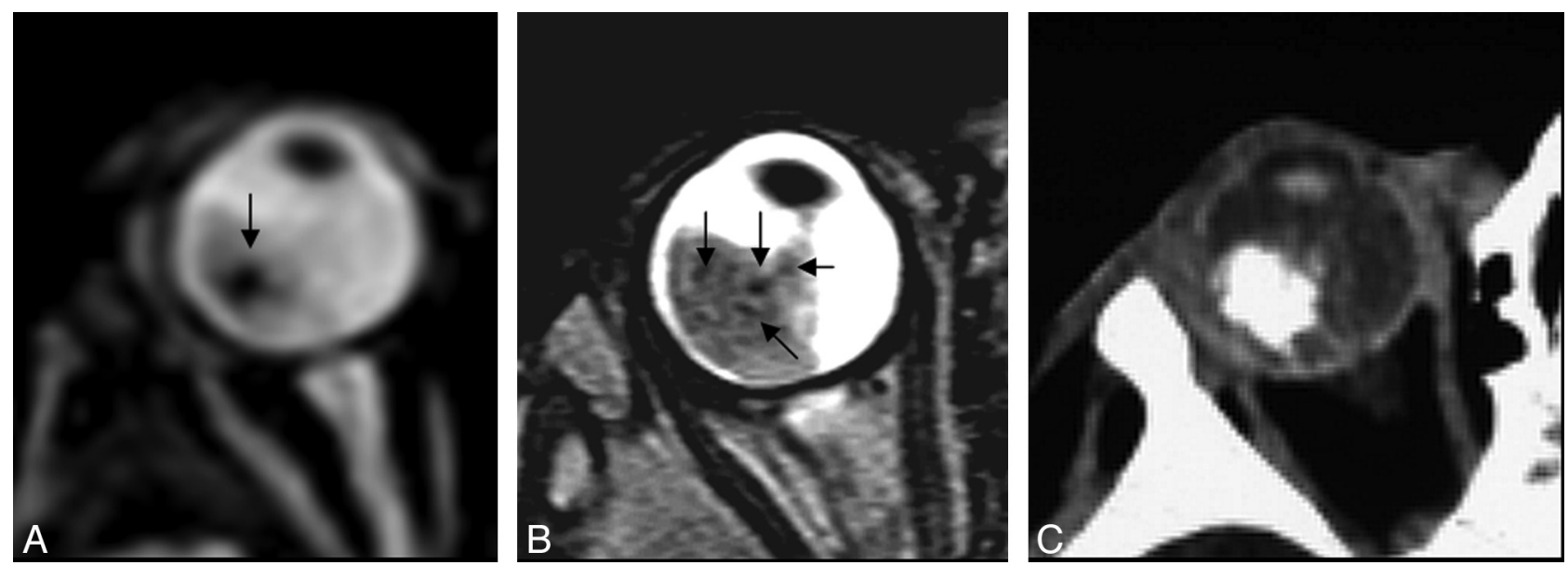

Fig 3. $A$, Focal areas of signal-intensity loss on a gradient-echo T2-weighted image (arrow in $A$ ) and a TSE T2-weighted image (arrows in B). C, CT scan confirms intralesional calcium deposits.

hemosiderin) and at the borders of melanocytic lesions. ${ }^{35}$ However, intratumoral hemorrhage in retinoblastoma is quite uncommon, whereas calcifications are present in a very high percentage of patients. Moreover, a real signal-intensity dropoff in gradient-echo images obtained on a high-field magnet is highly suggestive of calcifications, though hemosiderin or melanin may not be excluded; in our study, all areas suspected of calcifications at MR imaging were confirmed by CT examinations. Additionally, the correspondence between zones of signal-intensity loss on gradient-echo $\mathrm{T} 2^{*}$-weighted images and calcifications documented on CT scans was recently demonstrated in 4 patients with Fabry disease. ${ }^{38}$ Finally, no areas of hyperintensity on T2-weighted images (possibly corresponding to oxyhemoglobin and extracellular methemoglobin stages of hemorrhage) have been documented in our series, also confirmed by histology in the 28 enucleated bulbs. These data strengthen the hypothesis of the correlation between drop-off signal intensity on MR imaging and calcifications.

Despite the fact that retinoblastoma and melanoma usually have different ages of presentation, both the diseases may present in children older than 7 years of age and in teenagers (personal observation). ${ }^{13,39}$ The MR imaging characteristics of retinoblastoma are quite similar to those of uveal melanoma; however, to our knowledge, MR imaging in uveal melanoma 

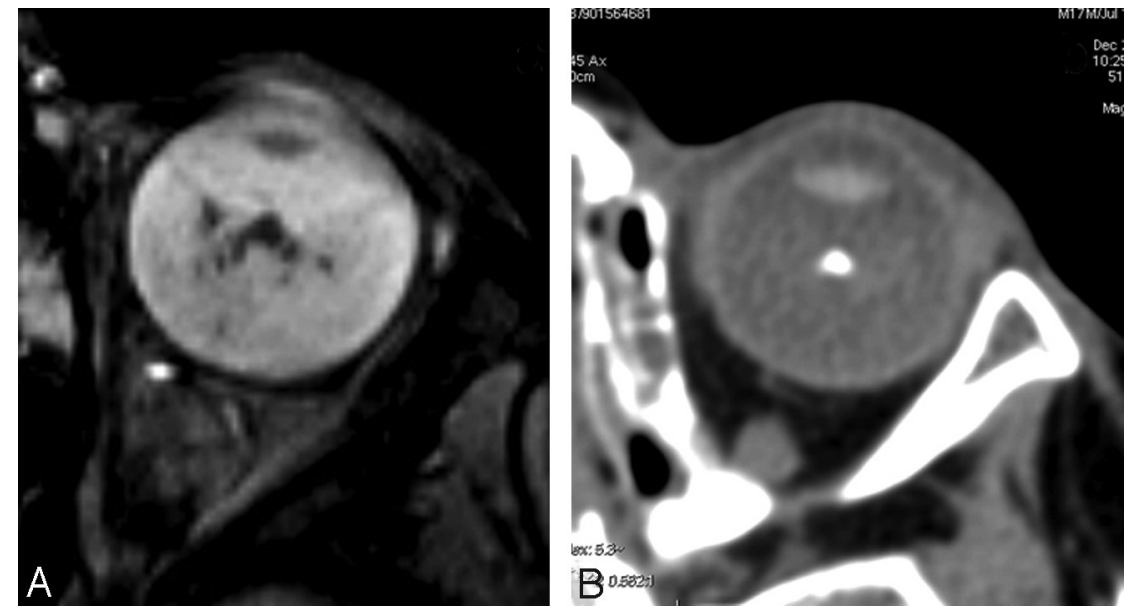

Fig 4. 3D gradient-echo T2-weighted image $(A)$ shows focal signal-intensity loss, corresponding to a hyperattenuating area on the CT scan $(B)$
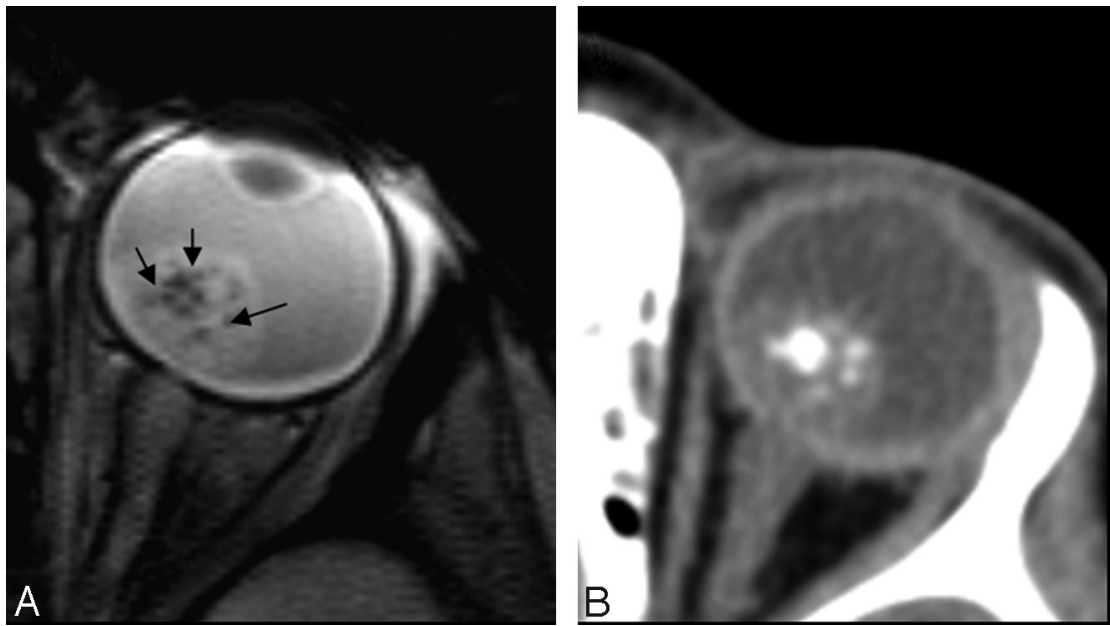

Fig 5. 3D gradient-echo T2-weighted image $(A)$ shows an even better depiction of small punctate calcifications $(a r r o w s, A)$ in comparison with the CT scan $(B)$

does not show spots of signal-intensity loss in spin-echo T2weighted and gradient-echo $\mathrm{T} 2^{\star}$-weighted imaging. Furthermore, we never noticed such a sign in our personal case records. Finally, proton MR spectroscopy may allow a differential diagnosis, because the visual appearance of retinoblastoma and uveal melanoma are completely different. ${ }^{11}$

Although CT is superior in calcium detection, ${ }^{40}$ the use of newer magnets (even if with the same magnetic field strength) allowing thinner sections may increase the specificity in calcium detection. We noticed that the use of very thin sections, both in TSE T2-weighted and in gradient-echo T2*-weighted techniques, may improve the detectability of intralesional spots. The development of a smaller diameter dedicated surface coil may allow the detection of very small intraocular lesions, even with a thickness of $<1 \mathrm{~mm} .^{27}$

Our study has clearly shown the possibility of detecting calcifications in retinoblastoma, when MR imaging, ultrasonographic, and ophthalmoscopic data are used jointly.

On the basis of the results of our study and considering the aims of orbital investigations in retinoblastoma, we think that a detailed neuroimaging study might comprise unenhanced T1-weighted, thin-section TSE T2, gradient-echo T2*weighted (when available, 3D sequences), and fat-suppressed enhanced T1-weighted images. Fat-suppressed enhanced T1- weighted images are preferable to (in comparison with) nonfat-suppressed spin-echo T1-weighted enhanced images because of the superiority in detecting optic nerve invasion and intraorbital extension of a retinoblastoma. ${ }^{41}$

\section{Conclusions}

Although intracranial calcifications have been extensively studied by MR imaging, there is not much detailed information about the capability of MR imaging in detecting intraocular calcifications.

We agree with the unquestionably superior specificity of CT in detecting calcifications; however, although brain calcification may be documented exclusively by means of neuroradiologic investigations, intraocular calcifications may also be discovered during ophthalmoscopic and ultrasonographic examination. In our 28 eyes with retinoblastoma, the combination of ophthalmologic investigations and MR imaging allowed us to make no mistakes in identifying calcifications; to the best of our knowledge, no study has coupled MR imaging and ophthalmologic investigations in detecting intraocular calcification.

We believe that if the ophthalmologist and the neuroradiologist work closely together, much more useful information on ocular structures may be obtained, including the detection 
of intraocular calcifications, without the use of potentially harmful ionizing radiation.

\section{Acknowledgments}

We thank Dr Donatella Balducci (Unit of Anesthesiology, Azienda Ospedaliera Senese) for her high-level experience in the sedation of children, and Drs Roberto Faleri and Ombretta Bugiani (University Library, Policlinico Le Scotte, Siena) for their invaluable help in finding pertinent literature manuscripts.

\section{References}

1. Singh AD, Shields CL, Shields JA. Prognostic factors in retinoblastoma. J Pediatr Ophthalmol Strabismus 2000;37:134-41

2. Karcioglu ZA, Gordon RA, Karcioglu GL. Tumor seeding in ocular fine-needle aspiration biopsy. Ophthalmology 1985;92:1763-67

3. Shields JA, Shields CL. Differentiation of Coats' disease and retinoblastoma. J Pediatr Ophthalmol Strabismus 2001;38:262-66

4. Roth DB, Scott IU, Murray TG, et al. Echography of retinoblastoma: histopathologic correlation and serial evaluation after globe-conserving radiotherapy or chemotherapy. J Pediatr Ophthalmol Strabismus 2001;38:136-43

5. Nicholson DH, Norton EWD. Diffuse infiltrating retinoblastoma. Trans Am Ophthalmol Soc 1980;78:265-89

6. Weber AL, Mafee MF. Evaluation of the globe using computed tomography and magnetic resonance. Isr J Med Sci 1992;23:145-52

7. Lemke A-J, Kazi I, Mergner U, et al. Retinoblastoma-MR appearance using a surface coil in comparison with histopathologic results. Eur Radiol 2007; $17: 49-60$

8. Beets-Tan RG, Hendricks MJ, Ramos LM, et al. Retinoblastoma: CT and MRI. Neuroradiology 1994;36:59-62

9. Char DH, Hedges TR, Norman D. Retinoblastoma: CT diagnosis. Ophthalmology 1984;91:1347-50

10. Lindahl S. Computed tomography of retinoblastoma. Acta Radiol Diagn (Stockh) 1986;27:513-18

11. Mafee MF, Goldberg MF, Cohen SB, et al. Magnetic resonance imaging versus computed tomography of leukocoric eyes and use of in vivo proton magnetic resonance spectroscopy of retinoblastoma. Ophthalmology 1989;96:965-75, discussion $975-76$

12. Edwards DP, Mafee MF, Garcia-Valenzuela E, et al. Coats' disease and persistent hyperplastic primary vitreous: role of MR imaging and CT. Radiol Clin North Am 1998;36:1119-31

13. Park JJ, Gole GA, Finnigan S, et al. Late presentation of a unilateral sporadic retinoblastoma in a 16-year-old girl. Aust N Z J Ophthalmolol 1999;27:365-68

14. De Potter P, Flanders AE, Shields JA, et al. The role of fat-suppression technique and gadopentetate dimeglumine in magnetic resonance imaging evaluation of intraocular tumors and simulating lesions. Arch Ophthalmol 1994;112:340-48

15. Smirniotopoulos JG, Bargallo N, Mafee MF. Differential diagnosis of leukokoria: radiologic-pathologic correlation. Radiographics 1994;14: 1059-79

16. De Potter P, Shields JA, Shields CL. Tumors and pseudotumors of the retina. In: De Potter P, Shields JA, Shields CL, eds. MRI of the Eye and Orbit. Philadelphia: J.B. Lippincott \& Company; 1995:93-116
17. Atlas SW, Galetta SL. The orbit and visual system. In: Atlas SW, ed. Magnetic Resonance Imaging of the Brain and Spine. 2nd ed. Philadelphia: Lippincott Williams \& Wilkins; 1996:1018-92

18. Barkhof F, Smeets M, van der Valk P, et al. MRI in retinoblastoma. Eur Radiol 1997;7:726-31

19. Kaufman LM, Mafee MF, Song CD. Retinoblastoma and simulating lesions. Radiol Clin North Am 1998;36:1101-17

20. Villablanca JP, Mafee MF, Kaufman LM, et al. Facies to remember: retinoblastoma, Coats' disease, and toxocariasis. Int J Neuroradiol 1998;4:41-50

21. Galluzzi P, Venturi C, Cerase A, et al. Coats' disease: smaller volume of the affected globe. Radiology 2001;221:64-69

22. Shields JA, Shields CL, Suvarnamani C, et al. Retinoblastoma manifesting as orbital cellulitis. Am J Ophthalmol 1991;112:442-49

23. Brisse HJ, Lumbroso L, Fréneaux PC, et al. Sonographic, CT, and MRI findings in diffuse infiltrative retinoblastoma: report of two cases with histologic comparison. AJNR Am J Neuroradiol 2001;22:499-504

24. Abramson DH. Retinoblastoma: diagnosis and management. CA Cancer J Clin 1982;32:130-40

25. Mafee FM, Mafee RF, Malik M, et al. Medical imaging in pediatric ophthalmology. Pediatr Clin N Am 2003;50:259-86

26. Wilms G, Marchal G, Van Fraeyenhoven L, et al. Shortcomings and pitfalls of ocular MRI. Neuroradiology 1991;33:320-25

27. Schueler AO, Hosten N, Bechrakis NE, et al. High-resolution magnetic resonance imaging of retinoblastoma. Br J Ophthalmol 2003;87:330-35

28. Ettl A, Krames J, Daxer A, et al. High-resolution magnetic resonance imaging of neurovascular orbital anatomy. Ophthalmology 1997;104:869-77

29. Davis PC, Hopkins KL. Imaging of the pediatric orbit and visual pathways: computed tomography and magnetic resonance imaging. Neuroimaging Clin N Am 1999;9:93-114

30. Duncan JL, Scott IU, Murray TG, et al. Routine neuroimaging in retinoblastoma for the detection of intracranial tumors. Arch Ophthalmol 2001;3:450-52

31. Henkelman RM, Watts JF, Kucharczyk W. High signal intensity in MR images of calcified brain tissue. Radiology 1991;179:199-206

32. Tsuruda JS, Bradley WG. MR detection of intracranial calcification: a phantom study. AJNR Am J Neuroradiol 1987;8:1049-55

33. Go JL, Zee CS. Unique CT imaging advantages: hemorrhage and calcification. Neuroimaging Clinics of North America 1998;8:541-52

34. Simon EM, Mc Caffery S, Rowley HA, et al. High-resolution 3D T2-weighted fast spin echo: new applications in the orbit. Neuroradiology 2003;45:489-92

35. Atlas SW, Grossman RI, Hackney DB, et al. Calcified intracranial lesions: detection with gradient-echo-acquisition rapid MR imaging. AJR Am J Roentgenol 1988;150:1383-89

36. Henkelman M, Kucharczyk W. Optimization of gradient-echo MR for calcium detection. AJNR Am J Neuroradiol 1994;15:465-72

37. Mihara F, Gupta KL, Joslyn JJ, et al. Intraocular hemorrhage and mimicking lesions: role of gradient-echo and contrast-enhanced MRI. Clin Imaging 1993;17:171-75

38. Moore DF, Ye F, Schiffmann R, et al. Increased signal intensity in the pulivinar on T1-weighted images: a pathognomonic MR imaging sign of Fabry disease. AJNR Am J Neuroradiol 2003;24:1096-101

39. Shields CL, Shields JA, Milite J, et al. Uveal melanoma in teenagers and children: a report of 40 cases. Ophthalmology 1991;98:1662-66

40. Kucharczyk W, Henkelman RM. Visibility of calcium on MR and CT: can MR show calcium that CT cannot? AJNR Am J Neuroradiol 1994;15:1145-48

41. Ainbinder DJ, Haik BG, Frei DF, et al. Gadolinium enhancement: improved MRI detection of retinoblastoma extension into the optic nerve. Neuroradiology 1996;38:778-78 